\title{
Preconditioning for Heterogeneous Problems
}

\author{
Sergey V. Nepomnyaschikh ${ }^{1}$ and Eun-Jae Park ${ }^{2}$
}

1 Institute of Computational Mathematics and Mathematical Geophysics, SD Russian Academy of Sciences, Novosibirsk, 630090, Russia. svnep@yonsei.ac.kr

2 Department of Mathematics, Yonsei University, Seoul 120-749, Korea.

ejpark@yonsei.ac.kr

Summary. The main focus of this paper is to suggest a domain decomposition method for mixed finite element approximations of elliptic problems with anisotropic coefficients in domains. The theorems on traces of functions from Sobolev spaces play an important role in studying boundary value problems of partial differential equations. These theorems are commonly used for a priori estimates of the stability with respect to boundary conditions, and also play very important role in constructing and studying effective domain decomposition methods. The trace theorem for anisotropic rectangles with anisotropic grids is the main tool in this paper to construct domain decomposition preconditioners.

\section{Introduction}

In order to present the basic idea of the algorithm, let us consider the following simple model problem. Let a domain $\Omega$ be the union of two non-overlapping subdomains which are rectangles, i.e., $\bar{\Omega}=\cup_{i=1}^{2} \bar{\Omega}_{i}$, where

$$
\Omega_{i}=\{(x, y) \mid i-1<x<i, 0<y<1\}, i=1,2 .
$$

In $\Omega$ we consider the following problem. Find $p$ such that

$$
\begin{aligned}
-\operatorname{div}(a \nabla p)=f & & \text { in } \Omega, \\
p=0 & & \text { on } \partial \Omega,
\end{aligned}
$$

where the matrix $a$ is given as follows: $a=\left(\begin{array}{cc}a^{x} & 0 \\ 0 & a^{y}\end{array}\right)$ with $a^{x}=a_{i}^{x}$ and $a^{y}=a_{i}^{y}$ being positive constants in each $\Omega_{i}, i=1,2$. Denote the interface by $\gamma=\partial \Omega_{1} \cup \partial \Omega_{2} \backslash \partial \Omega$.

For the problem (1) we introduce a flux variable, $u=-a \nabla p$, which is of interest in many applications. Writing $\alpha=a^{-1}(x)$, the inverse matrix of $a$, the problem $(1)$ is equivalent to seeking $(u, p)$ such that 


$$
\begin{aligned}
\alpha u+\nabla p=0 & \text { in } \Omega, \\
\operatorname{div} u=f & \text { in } \Omega, \\
p=0 & \text { on } \partial \Omega .
\end{aligned}
$$

Multiplying by test functions and integrating by parts we obtain the following weak formulation of problem (2)

$$
\begin{aligned}
(\alpha u, v)-(\operatorname{div} v, p) & =0, \quad \forall v \in H(\operatorname{div} ; \Omega), \\
-(\operatorname{div} u, q) & =-(f, q), \quad \forall q \in L^{2}(\Omega),
\end{aligned}
$$

where $H(\operatorname{div} ; \Omega)=\left\{v \in L^{2}(\Omega)^{2} \quad: \operatorname{div} v \in L^{2}(\Omega)\right\}$.

The variational formulation (3) fits the abstract framework that is generally used for mixed methods. It is well known that under the LBB condition the abstract framework is well-posed.

We consider the rectangular Raviart-Thomas mixed finite element spaces $V_{h} \subset H(\operatorname{div} ; \Omega)$ and $Q_{h} \subset L^{2}(\Omega)$ associated with the triangulation $\mathcal{T}_{h}$ of $\Omega$. The lowest order rectangular Raviart-Thomas elements are defined as follows:

$$
R T(T)=Q_{1,0}(T) \times Q_{0,1}(T), \quad Q(T)=Q_{0,0}(T) \quad \text { for rectangle } T .
$$

Define

$$
V_{h}=\left\{v \in H(\operatorname{div} ; \Omega):\left.v\right|_{T} \in R T(T), \quad \forall T \in \mathcal{T}_{h}\right\}
$$

and

$$
Q_{h}=\left\{q \in L^{2}(\Omega):\left.q\right|_{T} \in Q(T), \quad \forall T \in \mathcal{T}_{h}\right\} .
$$

For simplicity, we will consider the uniform rectangular decomposition $\mathcal{T}_{h}$, where mesh steps $h_{x}=\frac{1}{m}, h_{y}=\frac{1}{n}$ for some positive integers $m, n$ so that $\mathcal{T}_{h}=\mathcal{T}_{1 h} \cup \mathcal{T}_{2 h}$. For $i=1,2$

$$
\begin{aligned}
V_{h i} & =\left\{v \in H\left(\operatorname{div} ; \Omega_{i}\right):\left.v\right|_{T} \in R T(T), \quad \forall T \in \mathcal{T}_{i h}\right\}, \\
Q_{h i} & =\left\{q \in L^{2}\left(\Omega_{i}\right):\left.q\right|_{T} \in Q(T), \quad \forall T \in \mathcal{T}_{i h}\right\} .
\end{aligned}
$$

The standard mixed finite element approximation $\left(u_{h}, p_{h}\right) \in V_{h} \times Q_{h}$ is defined by

$$
\begin{aligned}
\left(\alpha u_{h}, v\right)-\left(\operatorname{div} v, p_{h}\right) & =0, \quad \forall v \in V_{h}, \\
-\left(\operatorname{div} u_{h}, q\right) & =-(f, q), \quad \forall q \in Q_{h} .
\end{aligned}
$$

Note that the normal component of the members in $V_{h}$ is continuous across the interior boundaries in $\gamma$. We relax this constraint on $V_{h}$ by introducing Lagrange multipliers; see Arnold and Brezzi [1985]. Let $E_{h}$ be the set of edges which belongs to $\gamma$. The Lagrange multipliers space $\Lambda_{h}$ to enforce the required continuity on $\gamma$ is defined by

$$
\Lambda_{h}=\left\{\mu \in L^{2}\left(\bigcup_{e \in E_{h}} e\right):\left.\left.\mu\right|_{e} \in V_{h} \cdot \nu\right|_{e} \text { for each } e \in E_{h}\right\}
$$


Then, the hybridized form of domain decomposition method for mixed finite elements is to find $\left(u_{i h}, p_{i h}, \lambda_{h}\right) \in V_{i h} \times W_{i h} \times \Lambda_{h}$ such that

$$
\begin{array}{r}
\sum_{i=1}^{2}\left(\alpha u_{i h}, v_{i}\right)-\sum_{i=1}^{2}\left(\operatorname{div} v_{i}, p_{i h}\right)+\sum_{i=1}^{2}<\lambda_{h}, v_{i} \cdot \nu_{i}>=0, \forall v_{i} \in V_{i h} \\
-\sum_{i=1}^{2}\left(\operatorname{div} u_{i h}, q_{i}\right)=-\left(f, q_{i}\right), \forall q_{i} \in W_{i h} \\
\sum_{i=1}^{2}<\mu, u_{i h} \cdot \nu_{i}>=0, \forall \mu \in \Lambda_{h} .
\end{array}
$$

With the standard ordering of the unknowns, the matrix equation for (6) is given by

$$
\left[\begin{array}{cccc}
A_{x} & 0 & B_{x}^{T} & C^{T} \\
0 & A_{y} & B_{y}^{T} & 0 \\
B_{x} & B_{y} & 0 & 0 \\
C & 0 & 0 & 0
\end{array}\right]\left[\begin{array}{c}
U_{x} \\
U_{y} \\
P \\
\Lambda
\end{array}\right]=\left[\begin{array}{c}
0 \\
0 \\
F \\
0
\end{array}\right] .
$$

\section{General Approach to Preconditioning Saddle Point Problems}

Let $V$ and $Q$ be Hilbert spaces. Let an operator $A: V \rightarrow V$ be linear, symmetric, positive definite, bounded and let a linear operator $B$ map $V$ into $Q$. Denote by $B^{T}$ the transpose operator for $B$. Let us consider the following saddle point problem: find $(u, p) \in V \times Q$ such that

$$
\mathcal{A} \chi:=\left[\begin{array}{cc}
A & B^{T} \\
B & 0
\end{array}\right]\left[\begin{array}{l}
u \\
p
\end{array}\right]=\left[\begin{array}{l}
g \\
f
\end{array}\right], g \in V, f \in Q .
$$

For the operator $\mathcal{A}$ we search for a preconditioner $R$ in the block-diagonal form

$$
R=\left[\begin{array}{cc}
A & 0 \\
0 & \Sigma
\end{array}\right]
$$

where $\Sigma$ maps $Q$ to $Q$.

Consider the spectral problem

$$
\mathcal{A} \chi=\lambda R \chi
$$

with $\Sigma=B A^{-1} B^{T}$. Then the eigenvalues of the problem belongs to the set

$$
\left\{\frac{1-\sqrt{5}}{2}, 1, \frac{1+\sqrt{5}}{2}\right\} \text {. }
$$

According to Rusten and Winther [1992] and Kuznetsov and Wheeler [1995], we have 
Lemma 1. Let $\hat{R}$ be a symmetric operator. If there are positive constants $c_{1}$ and $c_{2}$ such that

$$
c_{1}(R \chi, \chi) \leq(\hat{R} \chi, \chi) \leq c_{2}(R \chi, \chi), \quad \forall \chi \in V \times Q,
$$

then the eigenvalues $\lambda$ of $\hat{R}^{-1} \mathcal{A}$ belong to two segments

$$
\lambda \in\left[-d_{1},-d_{2}\right] \cup\left[d_{3}, d_{4}\right],
$$

where

$$
\begin{gathered}
d_{1}=\frac{1}{c_{1}}\left(\frac{\sqrt{5}-1}{2}\right), d_{2}=\frac{1}{c_{2}}\left(\frac{\sqrt{5}-1}{2}\right), \\
d_{3}=\frac{1}{c_{2}}, \quad d_{4}=\frac{1}{c_{1}}\left(\frac{\sqrt{5}+1}{2}\right) .
\end{gathered}
$$

To solve the problem $\mathcal{A} \chi=b$, we use the Lanczos method with a preconditioner $\hat{R}$ satisfying the lemma. Denote

$$
\theta=\frac{\max \left\{d_{1}, d_{4}\right\}}{\min \left\{d_{2}, d_{3}\right\}}, \quad q=\frac{\theta-1}{\theta+1} .
$$

Then, from general theory of iterative methods, if $\chi^{0}$ is an initial vector and $\chi^{n}$ is an approximation after $n$ iterations by the Lanczos method, the following estimate holds:

$$
\left\|\chi^{n}-\chi\right\|_{\hat{R}} \leq 2 q^{n}\left\|\chi^{0}-\chi\right\|_{\hat{R}}
$$

where

$$
\|\chi\|_{\hat{R}}=(\hat{R} \chi, \chi)^{\frac{1}{2}} .
$$

It means that the construction of an effective preconditioner for $\mathcal{A}$ has been reduced to the construction of an effective preconditioner for the Schur complement $B A^{-1} B^{T}$.

Remark 1. If the cost of the multiplication of $A^{-1}$ by a vector is small (for example if $\mathrm{A}$ is a diagonal matrix), then instead of solving the system with $\mathcal{A}$ by the Lanczos method, we can solve the system with the Schur complement $B A^{-1} B^{T}$ by a preconditioned conjugate gradient method.

\section{Preconditioning for the Schur complement}

Let us denote by $\hat{p}, \hat{q} \in R^{(n \cdot m+n+n \cdot m)}$ vectors in block form:

$$
\hat{p}=\left[\begin{array}{lll}
p_{1} & \lambda & p_{2}
\end{array}\right]^{T}, \quad \hat{q}=\left[\begin{array}{lll}
q_{1} & \mu & q_{2}
\end{array}\right]^{T}
$$

where

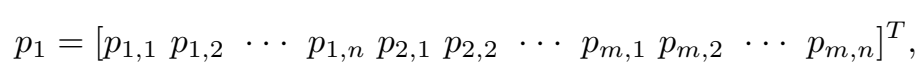




$$
\begin{aligned}
& \lambda=\left[\begin{array}{llll}
\lambda_{1} & \lambda_{2} & \cdots & \lambda_{n}
\end{array}\right]^{T},
\end{aligned}
$$

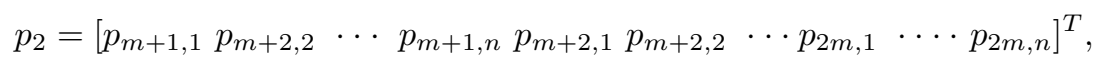

and $\hat{q}$ is similarly denoted.

Elimination of the flux variables in (7) reduces to the Schur complement which we denote by the matrix $S$ according to the ordering of unknowns $\left[\begin{array}{lll}p_{1} & \lambda & p_{2}\end{array}\right]^{T}$. Let

$$
\tilde{a}_{i}^{x}=a_{i}^{x} \frac{h_{y}}{h_{x}}, \quad \tilde{a}_{i}^{y}=a_{i}^{y} \frac{h_{x}}{h_{y}}, \quad i=1,2 .
$$

Then we define the $(n \cdot m+n+n \cdot m) \times(n \cdot m+n+n \cdot m)$ matrix $\tilde{S}$ so that

$$
\tilde{S} \hat{p}=\hat{q}
$$

where

$$
\begin{gathered}
-\tilde{a}_{1}^{x} p_{i-1, j}-\tilde{a}_{1}^{x} p_{i+1, j}-\tilde{a}_{1}^{y} p_{i, j-1}-\tilde{a}_{1}^{y} p_{i, j+1}+2\left(\tilde{a}_{1}^{x}+\tilde{a}_{1}^{y}\right) p_{i, j}=q_{i, j}, \\
i=1,2, \cdots, m, \quad j=1,2, \cdots, n, \\
-\tilde{a}_{2}^{x} p_{i-1, j}-\tilde{a}_{2}^{x} p_{i+1, j}-\tilde{a}_{2}^{y} p_{i, j-1}-\tilde{a}_{2}^{y} p_{i, j+1}+2\left(\tilde{a}_{2}^{x}+\tilde{a}_{2}^{y}\right) p_{i, j}=q_{i, j}, \\
i=m+1, m+2, \cdots, 2 m, \quad j=1,2, \cdots, n, \\
-\tilde{a}_{1}^{x} p_{m, j}-\tilde{a}_{2}^{x} p_{m+1, j}+\left(\tilde{a}_{1}^{x}+\tilde{a}_{2}^{x}\right) \lambda_{j}=\mu_{j}, \\
j=1,2, \cdots, n .
\end{gathered}
$$

Here

$$
\begin{aligned}
& p_{0, j}=0, \quad p_{2 m+1, j}=0, \quad j=1,2, \cdots, n, \\
& p_{i, 0}=0, \quad p_{i, n+1}=0, \quad i=1,2, \cdots, 2 m .
\end{aligned}
$$

The following is an analogue of Cowsar et al. [1995] and Kwak et al. [2003] for anisotropic case:

Lemma 2. There exist constant $c_{1}, c_{2}$, independent of $a^{x}, a^{y}, h_{x}, h_{y}$, such that for any $\hat{p}$

$$
c_{1}(S \hat{p}, \hat{p}) \leq(\tilde{S} \hat{p}, \hat{p}) \leq c_{2}(S \hat{p}, \hat{p}) .
$$

With the block representation of $\hat{p}$, we can consider a block form of $\tilde{S} \hat{p}$

$$
\begin{aligned}
\tilde{S} \hat{p} & =\left[\begin{array}{ccc}
B_{1} & B_{10} & 0 \\
B_{01}\left(B_{0}^{(1)}+B_{0}^{(2)}\right) & B_{02} \\
0 & B_{20} & B_{2}
\end{array}\right]\left[\begin{array}{c}
p_{1} \\
\lambda \\
p_{2}
\end{array}\right] \\
& =\left(\left[\begin{array}{ccc}
B_{1} & B_{10} & 0 \\
B_{01} & B_{0}^{(1)} & 0 \\
0 & 0 & 0
\end{array}\right]+\left[\begin{array}{ccc}
0 & 0 & 0 \\
0 & B_{0}^{(2)} & B_{02} \\
0 & B_{20} & B_{2}
\end{array}\right]\right)\left[\begin{array}{c}
p_{1} \\
\lambda \\
p_{2}
\end{array}\right] \\
& =\left(\tilde{S}_{1}+\tilde{S}_{2}\right) \hat{p} .
\end{aligned}
$$


According to the Additive Schwarz Method; see Matsokin and Nepomnyaschikh [1989], we can define a preconditioner $\tilde{\tilde{S}}$ for $\tilde{S}$ as

$$
\tilde{\tilde{S}}^{-1}=\left[\begin{array}{ccc}
\tilde{B}_{1}^{-1} & 0 & 0 \\
0 & 0 & 0 \\
0 & 0 & \tilde{B}_{2}^{-1}
\end{array}\right]+\left[\begin{array}{ccc}
0 & t_{1} & 0 \\
t_{1}^{T} & \Sigma^{-1} & t_{2}^{T} \\
0 & t_{2} & 0
\end{array}\right] .
$$

Here $\tilde{B}_{1}, \tilde{B}_{2}$ are spectrally equivalent to $B_{1}, B_{2}$ and $\Sigma$ is spectrally equivalent to the Schur complements for $\tilde{S}_{1}+\tilde{S}_{2}$ :

$$
\left(B_{0}^{(1)}-B_{01} B_{1}^{-1} B_{10}\right)+\left(B_{0}^{(2)}-B_{02} B_{2}^{-1} B_{20}\right)=\Sigma_{1}+\Sigma_{2}
$$

and $t_{1}, t_{2}$ extension operators of functions from $\gamma$ to $\Omega_{1}$ and $\Omega_{2}$ respectively such that

$$
\begin{aligned}
& c_{1}\left(\Sigma_{1} \lambda, \lambda\right) \leq\left(\tilde{S}_{1} t_{1} \lambda, t_{1} \lambda\right) \leq c_{2}\left(\Sigma_{1} \lambda, \lambda\right), \\
& c_{1}\left(\Sigma_{2} \lambda, \lambda\right) \leq\left(\tilde{S}_{2} t_{2} \lambda, t_{2} \lambda\right) \leq c_{2}\left(\Sigma_{2} \lambda, \lambda\right),
\end{aligned}
$$

for any $\lambda$.

For optimal convergence of the corresponding iterative method, all constants of spectral equivalence should be independent of $a^{x}, a^{y}, h_{x}, h_{y}$.

Now we consider only one subdomain $\Omega_{1}$. We omit subindex for the subdomain and denote by $\hat{p}$

$$
\hat{p}=\left[\begin{array}{ll}
p_{1} \lambda
\end{array}\right]^{T}
$$

with block vectors $p_{1}, \lambda$ defined as before and denote by $A_{0}$ the $n \times n$ matrix

$$
A_{0}=\left[\begin{array}{cccccc}
2 & -1 & & & & \\
-1 & 2 & -1 & & & \\
& & & & & \\
& & & & \\
& & -1 & 2 & -1 \\
& & & -1 & 2
\end{array}\right]
$$

and by $I$ the $n \times n$ identity matrix. Consider the following $(n \cdot m+n) \times(n \cdot m+n)$ matrix $\tilde{S}$

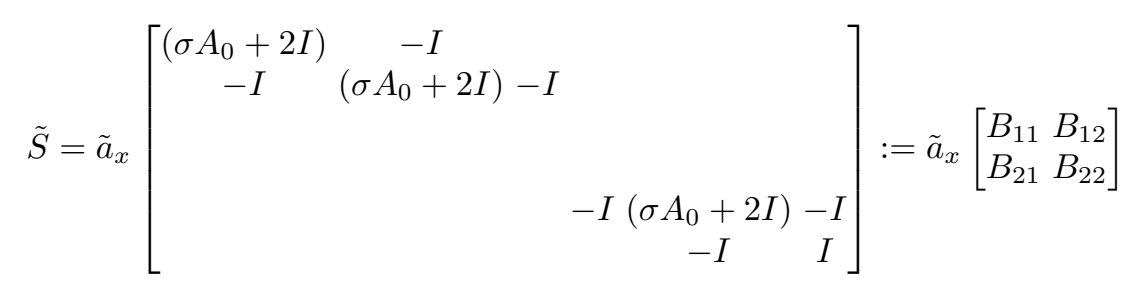

where

$$
\sigma=\frac{\tilde{a}^{y}}{\tilde{a}^{x}}, \quad \tilde{a}^{x}=a^{x} \frac{h_{y}}{h_{x}}, \quad \tilde{a}^{y}=a^{y} \frac{h_{x}}{h_{y}}, \quad B_{22}=I .
$$

Using the eigenvectors and eigenvalues of $A_{0}$ 
we have

$$
A_{0} q_{i}=\lambda_{i} q_{i}, i=1, \cdots, n
$$

$$
\begin{gathered}
q_{i}=\left[\begin{array}{c}
q_{i}(1) \\
q_{i}(2) \\
\cdot \\
\cdot \\
q_{i}(n)
\end{array}\right], \quad q_{i}(j)=\sqrt{\frac{2}{n+1}} \sin \frac{i \pi j}{n+1}, j=1,2, \cdots, n, \\
\lambda_{i}=4 \sin ^{2} \frac{\pi i}{2(n+1)},
\end{gathered}
$$

$$
\begin{gathered}
A_{0}=Q \Lambda Q^{T} \\
Q=Q^{T}=\left[q_{1}, \cdots, q_{n}\right], \quad \Lambda=\operatorname{diag}\left\{\lambda_{1}, \cdots, \lambda_{n}\right\} .
\end{gathered}
$$

To compute the Schur complement for $\tilde{S}$

$$
\Sigma=\tilde{a}^{x}\left(B_{22}-B_{21} B_{11}^{-1} B_{12}\right)
$$

we can use (8) and find a diagonal matrix $D$ such that

$$
\Sigma=Q D Q^{T}
$$

Using the technique from Matsokin and Nepomnyaschikh [1989], we have

Lemma 3. The diagonal matrix $D$ has the following elements.

$$
\begin{gathered}
D=\operatorname{diag}\left\{\mu_{1}(\Sigma), \mu_{2}(\Sigma), \cdots, \mu_{n}(\Sigma)\right\} \\
\mu_{i}(\Sigma)=\tilde{a}^{x}\left(1-\frac{U_{m-1}\left(\beta_{i}\right)}{U_{m}\left(\beta_{i}\right)}\right),
\end{gathered}
$$

where $\beta_{i}=\frac{1}{2} \sigma \lambda_{i}+1$ and $U_{j}$ is the Chebyshev polynomial of the second kind of degree $j$ so that

$$
U_{j}(x)=\frac{1}{2 \sqrt{x^{2}-1}}\left(\left(x+\sqrt{x^{2}-1}\right)^{j+1}-\left(x+\sqrt{x^{2}-1}\right)^{-(j+1)}\right) .
$$

Using the lemma for both subdomains $\Omega_{1}$ and $\Omega_{2}$, we can define

$$
\Sigma_{1}=Q D_{1} Q^{T} \text { for subdomain } \Omega_{1}
$$

and

$$
\Sigma_{2}=Q D_{2} Q^{T} \text { for subdomain } \Omega_{2}
$$

Then, put

$$
\Sigma=Q\left(D_{1}+D_{2}\right) Q^{T}
$$

and so

$$
\Sigma^{-1}=Q\left(D_{1}+D_{2}\right)^{-1} Q^{T} .
$$

Hence, finally we have the following theorem: 
Theorem 1. Let preconditioner $\tilde{\tilde{S}}$ be defined as

$$
\tilde{\tilde{S}}^{-1}=\left[\begin{array}{ccc}
\tilde{B}_{1}^{-1} & 0 & 0 \\
0 & 0 & 0 \\
0 & 0 & \tilde{B}_{2}^{-1}
\end{array}\right]+\left[\begin{array}{ccc}
0 & t_{1} & 0 \\
t_{1}^{T} & \Sigma^{-1} & t_{2}^{T} \\
0 & t_{2} & 0
\end{array}\right]
$$

with $\Sigma$ defined as above. Then, there exist constant $c_{1}, c_{2}$, independent of $a^{x}, a^{y}, h_{x}, h_{y}$, such that for any $\hat{p}$

$$
c_{1}(S \hat{p}, \hat{p}) \leq(\tilde{\tilde{S}} \hat{p}, \hat{p}) \leq c_{2}(S \hat{p}, \hat{p})
$$

To summarize, we have presented an optimal algorithm for a model anisotropic problem such that a condition number of the preconditioned problem is independent of parameters coefficients, grid sizes and the arithmetical cost of implementation of this algorithm is proportional to the number of degrees of freedom.

Acknowledgement. This work was supported by the Brain Korea 21 Project through Department of Mathematics, Yonsei University. EJP was supported in part by $\mathrm{Com}^{2} \mathrm{MaC}-\mathrm{KOSEF}$.

\section{References}

D. N. Arnold and F. Brezzi. Mixed and nonconforming finite element methods: implementation, postprocessing and error estimates. RAIRO Math. Model. Numer. Anal., 19:7-32, 1985.

L. C. Cowsar, J. Mandel, and M. F. Wheeler. Balancing domain decomposition for mixed finite elements. Math. Comp., 64(211):989-1015, July 1995.

Y. A. Kuznetsov and M. F. Wheeler. Optimal order substructuring preconditioners for mixed finite element methods on nonmatching grids. East-West J. Numer. Math., 3(2):127-143, 1995.

D. Kwak, S. Nepomnyaschikh, and H. Pyo. Domain decomposition for model heterogeneous anisotropic problem. Numer. Linear Algebra, 10:129-157, 2003.

A. M. Matsokin and S. V. Nepomnyaschikh. On using the bordering method for solving systems of mesh equations. Sov. J. Numer. Anal. Math. Modeling, 4:487-492, 1989.

T. Rusten and R. Winther. A preconditioned iterative method for saddle point problems. SIAM J. Matrix Anal., 13:887-904, 1992. 